\title{
Ecuaciones locales y generalizadas de altura-diámetro para pino radiata (Pinus radiata)
}

\author{
Local and generalized height-diameter equations for radiata pine (Pinus radiata)
}

\author{
Guillermo Trincado* y Cristián Leal D. \\ *Autor de correspondencia: Universidad Austral de Chile, Instituto de Manejo Forestal, Casilla 567, Valdivia, Chile, \\ Tel. (63) 221636, Fax. (63) 221231, gtrincad@uach.cl
}

\begin{abstract}
SUMMARY
In this study, two different approaches for modeling the height-diameter relationship were analyzed. Data were obtained from 3,492 sample plots established in 105 operational pre-harvest inventories in the province of Arauco, VIII Region. Measures of precision and bias permitted to evaluate and compare equations within and between modeling approaches. Moreover, a comparative analysis to determine the effect of total tree height prediction in stand volume and multi-product calculation was also investigated. The 2-parameter local equations were more precise and stable in terms of convergence than 3-parameter equations. Measures of precision and bias showed that generalized equations performed better than local equations. Paired $t$ tests $(\alpha=0.05)$ detected significant differences in total and merchantable volume estimates when applying a local or generalized equation. Contrarily, two of the three log products showed no significant difference.
\end{abstract}

Key words: height-diameter relationship, total height, radiata pine.

\section{RESUMEN}

Esta investigación analizó dos tipos de estrategias de modelamiento de la relación altura-diámetro (h/d) para ser utilizadas en labores de inventario. La información requerida se obtuvo de 3.492 parcelas temporales establecidas en 105 rodales de precosecha en la provincia de Arauco, VIII Región. El análisis consistió en una evaluación y comparación dentro y entre estrategias de modelamiento en base a medidas de precision y sesgo. Adicionalmente, se cuantificó el efecto en la estimación de alturas totales sobre la predicción de volúmenes y productos. Las ecuaciones locales con 2-parámetros fueron más precisas y estables en términos de convergencia en comparación a ecuaciones con 3-parámetros. En todas las evaluaciones realizadas las ecuaciones generalizadas presentaron mejores valores de precisión y sesgo que las ecuaciones locales. La aplicación de una prueba $t$ para observaciones pareadas $(\alpha=0,05)$ detectó diferencias significativas en la predicción de volúmenes totales y comerciales al utilizar ecuaciones locales o generalizadas. Sin embargo, dos de tres productos considerados no indicaron diferencias significativas.

Palabras clave: relación altura-diámetro, altura total, pino radiata.

\section{INTRODUCCIÓN}

En operaciones forestales una de las actividades más costosas en términos de tiempo y recursos es la medición de alturas totales en árboles en pie (Wang y Hann 1988, Dolph 1989, Zhang et al. 2002). Por esta razón una práctica normalmente utilizada ha sido el ajuste de ecuaciones altura-diámetro (h/d). La información de alturas es relevante debido a que es requerida en diversas actividades. En inventarios forestales, la utilización de alturas totales en conjunto con DAP permiten predecir con una mayor exactitud volúmenes totales y comerciales (Soares y Tomé 2002). El uso de sistemas de trozado destinados a predecir recuperación de productos están basados en ecuaciones de perfil fustal. Por lo tanto, su implementación también requiere conocer la altura total de cada individuo (Epstein et al. 1999). Prescripciones de manejo en plantaciones jóvenes están determinadas en gran medida por la productividad de sitio. Una estimación de la productividad de sitio se realiza a través del índice de sitio, que para su cálculo necesita conocer la edad y altura dominante de un rodal. Por otro lado, el análisis de parcelas permanentes en estudios de crecimiento y rendimiento requieren desarrollar ecuaciones de altura-diámetro en caso que mediciones periódicas de altura total no hubiesen sido realizadas (Assman 1970, Burkhart et al. 1972, Wang y Hann 1988, Huang et al. 1992, Lynch y Murphy 1995). Aun cuando también se han utilizado otros procedimientos para ajustar simultáneamente curvas de altura-diámetro para diferentes edades (Omule y MacDonald 1991, Flewelling y Jong 1994, Lappi 1997).

Probablemente el mayor uso de las ecuaciones h/d en el manejo de plantaciones ha sido en operaciones de inventarios forestales. Sin embargo, en Chile se ha desa- 
rrollado poca investigación con el propósito de identificar las ecuaciones más adecuadas para modelar la relación altura-diámetro en plantaciones (García 1974, Cox 1994). Por otro lado, actualmente existe una gran cantidad de formas funcionales y estrategias de modelamiento que han sido utilizadas en otras especies forestales (López Sánchez et al. 2003). Sin embargo, su utilidad aún no ha sido debidamente evaluada en plantaciones de pino radiata (Pinus radiata D. Don) en Chile.

La principal contribución de esta investigación es comparar diversas estrategias de modelamiento para predecir altura total en plantaciones de pino radiata manejadas intensivamente. Los objetivos específicos fueron (1) seleccionar las mejores ecuaciones locales y generalizadas de altura-diámetro, (2) evaluar la capacidad predictiva de las mejores ecuaciones dentro de cada una de las estrategias de modelamiento empleando medidas de precisión y sesgo, y (3) cuantificar el impacto de la predicción de altura total en el cálculo de volumenes totales y comerciales desagregados por productos.

\section{MÉTODOS}

Información dasométrica. La información necesaria se obtuvo de inventarios de precosecha realizados en plantaciones de pino radiata entre 1996-2001 por Forestal Mininco S.A. en la provincia de Arauco, VIII Región. Estas plantaciones fueron sometidas a intervenciones silviculturales de raleos y podas. Se seleccionaron rodales con una densidad no mayor a 800 árboles por hectárea con al menos $80 \%$ de los árboles podados (cuadro 1). El rango de superficies de los rodales fue entre 18-251 hectáreas y el número de unidades muestrales establecidas en forma sistemática dentro de cada uno de ellos fue entre 10-103 parcelas. Esto determinó finalmente una base muestral de 3.492 parcelas temporales establecidas en 105 rodales.

El tamaño de las parcelas establecidas fue entre 250$800 \mathrm{~m}^{2}$, donde se seleccionaron entre 3-8 árboles para efectuar una medición de altura total. En la submuestra se incluyeron árboles sin daño aparente y representativos de las clases diamétricas inferior, media y superior. La información de altura-diámetro fue subdividida aleatoria-
Cuadro 1. Estadística descriptiva de los 105 rodales de Pinus radiata utilizados en éste estudio.

Descriptive statistics for the 105 radiata pine stands used in the study.

\begin{tabular}{lccrc}
\hline Variables & Mínimo & Máximo & Media & $\mathrm{CV}(\%)^{\mathrm{b}}$ \\
\hline Índice de sitio (m) & \multicolumn{1}{c}{20,1} & 33,6 & 27,9 & 12,0 \\
Edad (años) & 19 & \multicolumn{1}{c}{24} & 20,4 & 6,3 \\
Árboles/ha & 216,7 & 796,7 & 490,8 & 28,3 \\
Área basal/ha & 20,1 & 58,9 & 37,9 & 18,9 \\
Árboles podados/ha & 215,9 & 782,5 & 471,2 & 29,4 \\
Altura de poda (m) & 2,8 & 10,1 & 5,5 & 21,2 \\
Altura total (m) & 18,4 & 34,3 & 26,4 & 13,9 \\
DAP (cm) & 22,5 & 46,3 & 32,7 & 14,8 \\
\hline
\end{tabular}

a Se utilizó una función de índice de sitio (edad de referencia 20 años) aportada por Mininco S.A.

b Coeficiente de variación.

mente dentro de cada rodal en dos diferentes bases de datos: $70 \%$ de la información $\left(\mathrm{n}_{1}=12.292\right.$ observaciones) fue utilizada para la estimación de parámetros de las ecuaciones (ajuste) y el 30\% restante $\left(\mathrm{n}_{2}=5.209\right.$ observaciones) se utilizó para el proceso de validación (cuadro 2). Las ecuaciones locales consideradas en el estudio fueron ajustadas en forma separada para cada uno de los rodales y las ecuaciones generalizadas considerando el total de datos de ajuste.

Modelamiento de la relación altura-diámetro. La relación altura-diámetro presenta comúnmente una forma sigmoidea con un punto de inflexión en la parte inferior de los datos o bien forma cóncava sin punto de inflexión aparente (Huang et al. 1992, Avery y Burkhart 2002). Yuancai y Parresol (2001) indican que un modelo biológicamente adecuado debiera presentar forma sigmoidea con incremento monotónico (dy/dt $>0)$, punto de inflexión $\left(\mathrm{d}^{2} \mathrm{y} / \mathrm{dt}=0\right.$, punto donde la curvatura cambia de signo $) \mathrm{y}$ un valor asintótico al aumentar el diámetro de los árboles. Un ejemplo de la relación entre altura total y DAP para el rodal № 48526 se presenta en la figura 1a. En este caso se observa una relación de forma cóncava, sin punto de inflexión aparente.

Cuadro 2. Estadística descriptiva de árboles utilizados en el proceso de ajuste $\left(\mathrm{n}_{1}=12.292\right)$ y validación $\left(\mathrm{n}_{2}=5.209\right)$. Descriptive statistics for samples trees used in the fitting $\left(n_{1}=12,292\right)$ and validation $\left(n_{2}=5,209\right)$ process.

\begin{tabular}{|c|c|c|c|c|c|c|c|c|}
\hline \multirow{2}{*}{ Variable } & \multicolumn{4}{|c|}{ Base de ajuste } & \multicolumn{4}{|c|}{ Base de validación } \\
\hline & Mínimo & Máximo & Media & $\mathrm{CV}(\%)$ & Mínimo & Máximo & Media & $\mathrm{CV}(\%)^{\mathrm{a}}$ \\
\hline DAP $(\mathrm{cm})$ & 10,0 & 64,6 & 32,5 & 27,2 & 11,0 & 64,5 & 32,7 & 27,3 \\
\hline Altura total (m) & 9,0 & 42,1 & 26,2 & 18,3 & 12,5 & 40,9 & 26,3 & 18,3 \\
\hline
\end{tabular}

a Coeficiente de variación. 

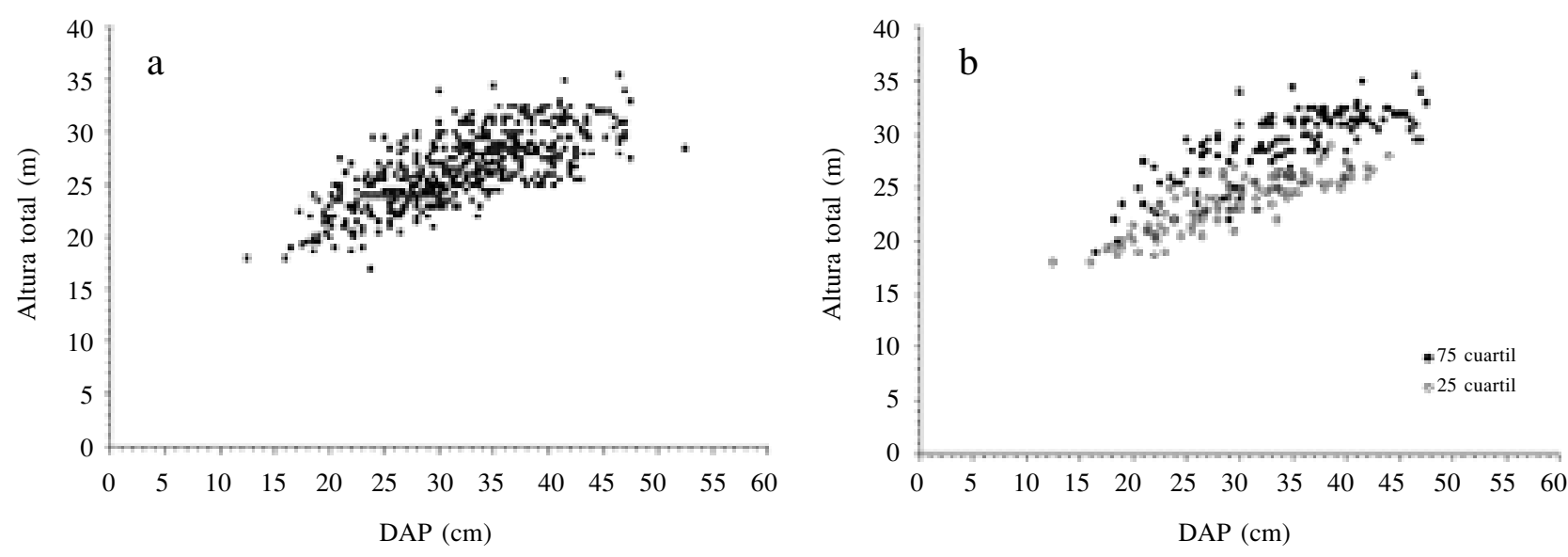

Figura 1. Relación de altura-diámetro (509 pares de observaciones) presentando (a) una forma de tipo cóncava y (b) altura total de árboles creciendo bajo diversos niveles de productividad (índice de sitio) dentro del rodal № 48526.

Height-diameter relationship (509 observations) showing (a) a concave shape and (b) the height of trees growing under different levels of productivity (site index) in stand No 48526.

La estimación de alturas totales para aquellos árboles no medidos puede ser realizado empleando ecuaciones locales o generalizadas de altura-diámetro. Otras metodologías que han sido propuestas recientemente incluyen el uso de distribuciones bivariadas $\mathrm{S}_{\mathrm{BB}}$ (Tewari y Gadow 1999, Dorado et al. 2001) y modelos de efectos mixtos (Hökkä 1997, Eerikäinen 2003).

Ecuaciones locales de altura-diámetro. Una práctica normal en operaciones forestales es ajustar una ecuación de altura-diámetro para cada rodal (Gadow y Hui 1999). Para esto es necesario seleccionar y medir dentro de cada parcela con la mayor exactitud posible una submuestra de árboles con el objetivo de obtener pares de observaciones de altura-diámetro. Arabatzis y Burkhart (1992) recomiendan el uso de muestreo aleatorio simple sin reemplazamiento durante el proceso de selección de árboles muestras para desarrollar este tipo de ecuaciones. Posteriormente, esta información se utiliza para ajustar una ecuación local de altura-diámetro (van Laar 1986, Huang et al. 2000). Este tipo de ecuaciones presentan la siguiente forma general:

$$
h_{i}=f\left(d_{i}, \beta\right)+\varepsilon_{i} i=1, \ldots, n
$$

donde $h_{i}$ es la altura total, $d_{i}$ es el diámetro a la altura de pecho (DAP) con corteza, $\beta$ es el vector de parámetros a ser estimado y $\varepsilon_{i} \sim \mathrm{N}\left(0, \sigma^{2}\right)$ es un error aleatorio que presenta una distribución de tipo normal. Inicialmente las curvas de altura-diámetro se generaron utilizando métodos gráficos, siendo luego reemplazados por técnicas de análisis de regresión (Nash 1965, Curtis 1967). Las primeras ecuaciones publicadas para estimar altura total presentaron formas lineales en los parámetros (Curtis 1967, Wang y Hann 1988). Sin embargo, estudios posteriores han demostrado que ecuaciones no lineales pueden modelar con mayor exactitud la relación altura-diámetro (Leroy 1989, Zhang 1997, Peng et al. 2001); aun cuando ciertas formas de ecuaciones de tipo lineal han seguido siendo recomendadas (García 1996, Woollons 2003). Desde un punto de vista biológico las ecuaciones no lineales aseguran un comportamiento más consistente de la curva, especialmente cuando se requiren efectuar predicciones fuera del rango de datos h/d usados en el ajuste (Huang et al. 1992). En esta investigación el interés fue analizar y comparar exclusivamente ecuaciones de tipo no lineal que han demostrado ser adecuadas para otras especies forestales (cuadro 3).

Las ecuaciones locales consideradas presentaron dos o tres parámetros. Además, algunas de ellas han sido condicionadas para estimar una altura total igual a $1,3 \mathrm{~m}$ cuando el DAP es igual a cero (por ejemplo, ecuaciones E6 y E9). Esta restricción es razonable desde un punto de vista teórico, aun cuando raras veces se está interesado en la predicción de alturas totales en árboles con DAP cercanos a cero (Fang y Bailey 1998).

Ecuaciones generalizadas de altura-diámetro: Dentro de las ecuaciones para modelar la relación altura-diámetro se han incluido variables a nivel de rodal con el objetivo de mejorar la capacidad predictiva (Larsen y Hann 1987, Krumland y Wensel 1988, Wang y Hann 1988, Gaffrey 1988, Dolph 1989, Bi et al. 2000, López et al. 2003). Por otro lado, la incorporación de variables a nivel de rodal permiten una aplicación más extensiva en comparación a ecuaciones de tipo local. Este tipo de ecuaciones son conocidas como ecuaciones generalizadas o regionales de altura-diámetro (Krumland y Wensel 1988, Wenk et al. 1990, Soares y Tomé 2002). 
Cuadro 3. Ecuaciones locales y generalizadas de altura-diámetro seleccionadas para el estudio. Selected local and generalized height-diameter equations for this study.

Forma de la ecuacióna

$$
\begin{aligned}
& \text { Ecuaciones locales de altura diametro } \\
& \text { E.: } \quad h=1,3+\beta_{0} e^{\beta_{1} d^{-1}}+\varepsilon \\
& \text { F2: } \quad h=1,3+e^{\beta_{a}+\frac{\varepsilon_{1}}{1+d}}+\varepsilon \\
& \text { E3: } \quad h=1,3+A_{0}\left(\frac{d}{1+d}\right)^{h}+\varepsilon \\
& \text { E4: } \quad h=\beta_{0} e^{\delta \delta^{-1}}+\varepsilon \\
& \text { E5. } h=1,3+\left(\beta_{0}+\beta_{1} d^{=1}\right)^{-13}+\varepsilon \\
& \text { E6: } h=1,3+\beta_{0}\left(1-e^{-\beta_{1} d}\right)^{\beta_{2}}+\varepsilon \\
& \text { E7: } h=1,3+\beta^{2}+A d^{2}+\varepsilon \\
& \text { Ex } \quad h=1,3+\beta_{0} e^{-\beta_{1} d^{-\lambda_{2}}}+\varepsilon \\
& \text { E9: } h=1,3+\beta_{\mathrm{a}}\left(1-\mathrm{e}^{-\beta_{1} d^{f_{2}}}\right)+e \\
& \text { Et0: } h=1.3+\beta_{0} \pi 1+1 /\left(\beta_{1} d^{\beta_{2}} \|+\varepsilon\right.
\end{aligned}
$$

Eeuaciones gerneralizadas de aftura-dismetro

EIt: $\quad h=1,3+\left[\beta_{b}\left(1 / d-1 / / D_{d}\right)+\left(1 /\left(/ H_{d}-1,3\right)\right)^{1 / 3}\right]^{-3}+c$

$$
\begin{aligned}
& \text { EI2: } h=1,3+\left(H_{d}-1,3\right) e^{\beta_{i}\left(1-\frac{D_{2}}{d}\right)+\beta_{i}\left(\frac{1}{\theta_{6}}-\frac{1}{d}\right)}+\sigma \\
& \text { E.13: } \left.h=H_{\mathrm{d}} e^{-\beta_{0}\left(d^{-\left(\sigma_{1}+s_{2} H_{d}\right)}-D_{d}-\left(s_{1}+\beta_{2} H_{d}\right)\right.}\right)+\varepsilon \\
& \text { E14: } \quad h=1,3+\left(\beta_{0}+\beta_{1} H_{4}+\beta_{2} D_{8}\right) e^{-\beta_{3} / d}+\varepsilon \\
& \text { E15: } \quad h=1.3+\mathrm{e}^{\beta_{0}+\beta_{1} d \rho_{2}+\beta_{3} S}+6 \\
& \text { E16: } \quad h=1,3+\beta_{a} H_{d}^{\beta_{1}} d{ }^{\beta_{2} \mu_{d}^{\beta_{3}}}+\epsilon \\
& \text { EI7: } \quad h=\beta_{0} H_{d}\left[1-\left.\beta_{1} e^{-\beta_{2} d / D_{2}}\right|^{\beta_{3}}+\varepsilon\right. \\
& \text { E18: } h=1,3+e^{\beta_{0}+\beta_{1} \delta^{2} 2+\beta_{3} s+\beta_{4} \ln (C)}+\delta
\end{aligned}
$$

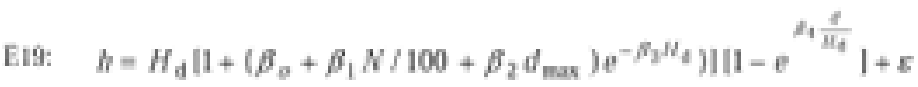

Ejemplos de aplicación

Fewelling y Jong (1994), Martin y Flewelling (1995). Haks (1997)

Wyboff ed al (1982). Dolph (1989). Moore ef al (1969)

Prodan (1965), Curtis (1967), Huang ef al. (1992), Martin y Fiewelling (1958)

Mraharzis y Burkhan (1992)

Woollons (2003)

Hang of al, (1992), Huang y Titus (1994). Zhang of at. (1995), Zhang (1997), Zlang ef a.' (2002)

larsen y Hann (1985). Wang y Hann (1988). Dolph (1989). Panresol (1992)

Zeide (1960). Zhang ef al (1995). Moore o al. (1506)

Yang ef al (1978). Perget al (z001)

Hang of at. $(2000)$

Omule y MacDonald (1991)

Gaffrey [1968). Nagel [1991]. Sloboda of al (1993). Hui y von Gadow (1990), van 1 aar y Akga (1997)

Krumland y Wrnsel (1968)

Hol y Cadow (1993). Prodan erall (1997, p. 325)

Larsen y Hann (1987). Wang y llann (1988)

Ihat y Gadow (1993)

Hul y Gadow (1993): Schuöder y Alvarex Conzllez (2001)

Dolph (1960)

Souses y Tomb [2002), Lopex Sanclez ef al. [2000]

a $h=$ altura total $(\mathrm{m}) ; d=$ DAP con corteza a una altura de $1.3 \mathrm{~m}(\mathrm{~cm}) ; d_{\max }=$ máximo DAP $(\mathrm{cm}) ; D_{\mathrm{g}}=$ diámetro medio cuadrático $(\mathrm{cm}) ;$ $D_{\mathrm{d}}=$ DAP medio de árboles dominantes; $H_{\mathrm{d}}=$ altura dominante (altura media de los 100 árboles con los mayores DAP por hectárea); $S=$ índice de sitio (m) a una edad de referencia de 20 años; $N=$ número de árboles por hectárea; $G=$ área basal por hectárea $\left(\mathrm{m}^{2}\right.$ ha $\left.{ }^{-1}\right) ;$ $\mathrm{b}_{i}=$ parámetros a ser estimados $(i=1,2, \ldots, \mathrm{n}) \quad \mathrm{y} \mathrm{e}=$ error aleatorio. 
Las variables a nivel de rodal incluidas frecuentemente son el índice de sitio $(S)$ y área basal $(G)$. Ambas variables son de importancia debido al efecto que tienen sobre la relación altura-diámetro (Wang y Hann 1988). Dentro de un rodal se ha observado que para un mismo DAP se alcanza una mayor altura total bajo mejores condiciones de sitio (Huang et al. 1992, Prodan et al. 1997). Como una manera de verificar esta observación se estimó el índice de sitio para cada una de las 102 parcelas del rodal № 48526. Posteriormente se seleccionaron todos aquellos pares de observaciones altura-diámetro contenidas en el 25 y 75 cuartil de la distribución de índice de sitio (figura 1b). Acá se observa claramente que para un mismo DAP, mejores condiciones de sitio favorecen el crecimiento en altura total.

Las ecuaciones generalizadas utilizadas en este estudio fueron ajustadas considerando la totalidad de datos (cuadro 3). Por lo tanto, antes de realizar el ajuste se requirió calcular en cada parcela las siguientes variables: diámetro medio de árboles dominantes $\left(D_{\mathrm{d}}\right)$, diámetro medio cuadrático $\left(D_{\mathrm{g}}\right)$, máximo diámetro $\operatorname{DAP}\left(d_{\max }\right)$, número de árboles por hectárea $(N)$, altura dominante $\left(H_{\mathrm{d}}\right)$ e índice de sitio $(S)$. La altura dominante $\left(H_{\mathrm{d}}\right)$ se definió como el promedio de alturas de los 100 árboles de mayores DAP por hectárea. Para calcular la altura dominante en cada parcela se debieron predecir aquellas alturas de árboles dominantes que no fueron medidas. El procedimiento utilizado fue ajustar y utilizar para cada rodal la ecuación local de altura-diámetro E1 (cuadro 3). A partir del cálculo de la altura dominante dentro de cada parcela y la edad del rodal se estimó el índice de sitio con una función empleada por Mininco S.A. Una mejor estimación de altura dominante dentro de cada parcela hubiese sido posible midiendo la altura total de cada árbol dominante. Sin embargo, el escaso número de mediciones de altura-diámetro dentro de cada parcela limitaron las posibilidades de estimación.

El objetivo de calcular altura dominante e índice de sitio (al igual que otras variables) a nivel de parcela fue para permitir una mejor caracterización de las condiciones locales de crecimiento dentro de cada rodal (18-251 hectáreas).

Ajuste y comparación de las ecuaciones. La estimación de parámetros para cada una de las ecuaciones se realizó utilizando la subrutina PROC NLIN contenida en el software estadístico Statistical Analysis System (SAS Institute 1999). Durante el proceso de ajuste el algoritmo de minimización de la suma de cuadrados del error fue MARQUARDT (Zhang 1997, Peng et al. 2001). La calidad de ajuste de cada una de las ecuaciones fue comparada utilizando el error estándar de estimación $\left(\mathrm{s}_{y . x}\right)$

$$
\mathrm{s}_{y . x}=\sqrt{\sum_{i=1}^{n}\left(h_{i}-\hat{h}_{i}\right)^{2} /(n-p)},
$$

donde $h_{i}$ es la altura total observada, $\hat{h}_{i}$ es la altura total estimada, $n$ es el número total de observaciones y p es el número de parámetros de la ecuación. La significancia de los coeficientes se evaluó utilizando una prueba $t$ $(\alpha=0,05)$. Los residuales de cada ecuación se analizaron gráficamente para evaluar normalidad y homogeneidad de varianza, esto con el propósito de obtener una estimación insesgada de parámetros (Myers 1990). En caso de existir heterogeneidad de varianza, se consideró la aplicación de factores de ponderación. El supuesto de normalidad de residuales fue evaluado también gráficamente utilizando Q-Q plots.

Capacidad predictiva de las ecuaciones seleccionadas. En el proceso de validación se consideraron sólo las cuatro mejores ecuaciones dentro de cada una de las estrategias de modelamiento (cuadro 3). La selección preliminar de ecuaciones se basó en los siguientes criterios: (i) estadística de ajuste, (ii) número de rodales para los cuales el algoritmo de minimización MARQUARDT presentó convergencia en la estimación de parámetros para el caso de ecuaciones locales, (iii) significancia de los parámetros estimados y (iv) número de parámetros y variables predictoras contenidas en la ecuación. Posteriormente se evaluó la capacidad predictiva de cada ecuación seleccionada calculando medidas de precisión (REMC, raíz del error medio cuadrático)

$$
\mathrm{REMC}=\sqrt{\frac{\sum_{i=1}^{m}\left(h_{i}-\hat{h}_{i}\right)^{2}}{m}} \quad i=1, \ldots, m
$$

y sesgo (DM, diferencia media)

$$
\mathrm{DM}=\frac{\sum_{i=1}^{m}\left(h_{i}-\hat{h}_{i}\right)}{m} \quad i=1, \ldots, m
$$

considerando el total de datos de validación (Allen et al. 1992). Bajo los criterios utilizados las mejores ecuaciones fueron aquellas que presentaron una alta precisión y bajo sesgo. Esto es equivalente a obtener bajos valores absolutos para la REMC y DM. Por otro lado, valores positivos de DM indicaron subestimación y valores negativos sobreestimación. Adicionalmente, para cada uno de los estadísticos, se calcularon valores porcentuales dividiendo el valor obtenido por la altura media observada en la base de datos de validación.

Un análisis complementario consideró calcular en cada uno de los rodales la REMC para cada una de las ocho ecuaciones seleccionadas (105 rodales). El sistema de evaluación consistió en asignar un ranking para cada ecuación dentro de cada rodal. La ecuación que presentó 
el menor REMC (mayor precisión) en un determinado rodal le fue asignado el ranking uno, el segundo menor valor ranking dos, y así sucesivamente. El tipo de información obtenida fue el número de veces en la cual cada ecuación presentó un determinado ranking (1-8) para un total de 105 rodales. Este análisis permitió identificar la mejor ecuación (dentro y entre estrategias de modelamiento) comparando el grado de precisión en las predicciones.

Efecto de la predicción de alturas en el cálculo de volúmenes y productos. Cada rodal fue procesado en dos oportunidades utilizando una ecuación local y una ecuación generalizada. Esto con el fin de determinar si diferentes métodos de predicción de altura total tienen un efecto importante en el cálculo de volúmenes totales y productos. Los volúmenes y productos posibles de obtener de cada árbol se estimaron utilizando una ecuación de perfil fustal de forma polinomial (Bruce et al. 1968). Esta ecuación fue previamente ajustada para pino radiata por Martin (1993). En la obtención de productos, la secuencia de corte a lo largo del fuste fue determinada por la prioridad asignada a cada producto dentro del esquema de trozado (cuadro 4).

El algoritmo de corte parte de la base fustal asumiendo una altura de tocón residual de $0,3 \mathrm{~m}$. Posteriormente éste trata de maximizar el número de trozas del tipo AS01 de acuerdo a las restricciones de dimensiones establecidas (cuadro 4). Cuando no es posible la obtención de un mayor número de trozas AS01, el algoritmo continúa la secuencia de corte considerando la siguiente prioridad asignada (AS02) y sus restricciones de dimensiones asociadas. El mismo procedimiento continúa iterativamente hasta alcanzar un diámetro mínimo comercial de $10 \mathrm{~cm}$ (pulpa), en donde no es posible obtener más trozas aprovechables industrialmente.
Cuadro 4. Esquema de trozado y especificación de productos. Bucking pattern and specification of log-products dimensions.

\begin{tabular}{lcccl}
\hline Producto & Prioridad & $\begin{array}{c}\text { Largo } \\
(\mathrm{m})\end{array}$ & $\begin{array}{c}\text { Diámetro } \\
\text { mínimo } \\
(\mathrm{cm})\end{array}$ & $\begin{array}{c}\text { Destino } \\
\text { industrial }\end{array}$ \\
\hline AS01 & 1 & 5,50 & 26 & Aserradero \\
AS02 & 2 & 4,10 & 18 & Aserradero \\
Pulpa & 3 & 2,44 & 10 & Pulpa \\
\hline
\end{tabular}

La determinación de diferencias en la estimación de volúmenes bajo diferentes estrategias de predicción de altura total se evaluó aplicando una prueba $t$ para observaciones pareadas $(\alpha=0,05)$. La hipótesis nula $\left(\mathrm{H}_{0}: \mu_{0}-\mu_{1}=0\right)$ indica que la diferencia media en volumen utilizando diferentes métodos de predicción es igual a cero. Por lo tanto, el no rechazo de $\mathrm{H}_{0}$ señalaría que no existe evidencia concluyente para inferir que el empleo de diferentes tipos de ecuaciones de altura-diámetro tienen un efecto significativo sobre la estimación de volúmenes.

\section{RESULTADOS}

Parámetros estimados de las ecuaciones. Un análisis gráfico de residuales estudentizados para cada una de las ecuaciones locales no indicaron la presencia de heterogeneidad de varianza (heterocedasticidad). Por lo tanto, no se utilizaron factores de ponderación durante el proceso de ajuste. Los residuales en todas las ecuaciones también presentaron una distribución de tipo normal. Los parámetros medios estimados y calidad de ajuste de cada una de las ecuaciones se presentan en el cuadro 5.

Cuadro 5. Parámetros medios estimados, error estándar de estimación $\left(\mathrm{s}_{y . x}\right)$ y número de veces que el algoritmo de minimización MARQUARDT converge en la estimación de parámetros en cada uno de los 105 rodales.

Estimated mean parameters, standard error of estimate $\left(s_{y . x}\right)$ and number of times that the Marquardt algorithm converged in estimating the equation parameters for each of the 105 sampled stands.

\begin{tabular}{|c|c|c|c|c|c|c|c|}
\hline \multirow{2}{*}{ Ecuación } & \multicolumn{3}{|c|}{ Parámetros medios } & \multicolumn{3}{|c|}{$\mathrm{s}_{y . x}(\mathrm{~m})$} & \multirow{2}{*}{ Convergencia } \\
\hline & $\hat{\beta}_{0}$ & $\hat{\beta}_{1}$ & $\hat{\beta}_{2}$ & Mínimo & Máximo & Media & \\
\hline E1 & 36,052 & $-11,098$ & - & 0,80 & 3,52 & 1,91 & 105 \\
\hline E2 & 3,591 & $-11,936$ & - & 0,80 & 3,51 & 1,91 & 105 \\
\hline E3 & 36,311 & 11,510 & - & 0,80 & 3,51 & 1,91 & 105 \\
\hline E4 & 37,209 & $-10,508$ & - & 0,80 & 3,52 & 1,91 & 105 \\
\hline E5 & 0,236 & 1,253 & - & 0,82 & 3,53 & 1,92 & 105 \\
\hline E6 & 32,410 & 0,061 & 1,428 & 1,12 & 3,32 & 1,93 & 89 \\
\hline E7 & 3,834 & $-119,308$ & $-1,115$ & 1,11 & 3,32 & 1,93 & 88 \\
\hline E8 & 47,810 & 124,746 & 1,161 & 1,11 & 3,32 & 1,91 & 84 \\
\hline E9 & 33,636 & 0,058 & 1,033 & 1,12 & 3,31 & 1,93 & 89 \\
\hline E10 & 47,544 & 0,027 & 1,495 & 1,11 & 3,32 & 1,92 & 92 \\
\hline
\end{tabular}


En base a los valores medios de error estándar de estimación $\left(s_{y . x}\right)$ no se observaron mayores diferencias entre las ecuaciones. Sin embargo, las ecuaciones con 2-parámetros presentaron comparativamente menores errores. En algunos rodales las ecuaciones con 3-parámetros no pudieron ser ajustadas a los datos. Esto debido a que el algoritmo MARQUARDT no pudo determinar valores de parámetros que garanticen un mínimo global. Por ejemplo, la ecuación E6 se ajustó en sólo 89 de un total de 105 rodales. Comparativamente, las ecuaciones con 2-parámetros ajustaron en todos los rodales. Por lo tanto, los modelos seleccionados en esta etapa para validar la capacidad predictiva fueron las ecuaciones de 2-parámetros E1, E2, E3 y E4.

En todas las ecuaciones generalizadas la varianza de los residuales fue homogénea y su distribución de tipo normal. El error estándar de estimación $\left(s_{\mathrm{y} . \mathrm{x}}\right)$ indicó que no existen mayores diferencias entre las ecuaciones (cuadro 6).

Sin embargo, en dos ecuaciones (E16 y E17) se detectaron parámetros que no fueron significativamente diferentes de cero. De acuerdo a los criterios establecidos se seleccionaron las ecuaciones E11, E12, E13 y E14 para evaluar su capacidad predictiva.

Predicción dentro y entre estrategias de modelamiento. Las ecuaciones generalizadas de altura-diámetro presentaron consistentemente mayor precisión (menores valores en REMC) que las ecuaciones locales (figura 2a). Por otro lado, el sesgo fue variable dentro de cada una de las estrategias de modelamiento presentando en todos los casos valores absolutos menores a 0,2 m (figura 2b). Dentro de las ecuaciones locales, la ecuación E3 presentó notoriamente el mayor sesgo, mientras que las restantes presentaron sesgos similares (E1, E2 y E4). En todos los casos las ecuaciones locales subestimaron los valores observados. Comparativamente las ecuaciones generalizadas presentan menores sesgos, pero con una mayor variabilidad de valores entre ellas. En general las ecuaciones generalizadas subestimaron los valores observados, salvo la ecuación E13.

El cuadro 7 presenta el número de veces que una determinada ecuación recibió un determinado ranking para la totalidad de rodales. Por ejemplo, dentro de 105 rodales la ecuación E3 presentó ranking uno en cuatro oportunidades, ranking dos en dos oportunidades, etc. Las ecuaciones generalizadas estuvieron localizadas mayoritariamente en los cuatro primeros lugares y las ecuaciones locales en los últimos cuatro lugares del ranking. Esto determinó que el ranking promedio para las ecuaciones locales fue de 5,96 y para las ecuaciones generalizadas de 2,98. Esta observación se consideró como una indicación que ecuaciones generalizadas presentan una mayor precisón que las ecuaciones locales.

Dentro de las ecuaciones locales, las ecuaciones E1 y E4 presentaron los mejores valores dentro del ranking establecido. La única diferencia existente entre ellas es que E1 está condicionada a entregar un valor de altura total igual a 1,3 m cuando el DAP se aproxima a cero. Comparativamente todas las ecuaciones generalizadas presentaron valores muy similares en cuanto al ranking promedio (cuadro 7). Sin embargo, las ecuaciones E12 y E13 aparecieron con mayor frecuencia en los mejores rankings, por lo que fueron consideradas ser las mejores ecuaciones.

Estimación volúmenes totales, comerciales y productos. Las ecuación local E4 (Arabatzis y Burkhart 1992) y la ecuación generalizada E13 (Krumland y Wensel 1988) fueron utilizadas en el cálculo de volúmenes y productos

Cuadro 6. Parámetros estimados y error estándar de estimación $\left(s_{\mathrm{y} . \mathrm{x}}\right)$ para las ecuaciones generalizadas de altura-diámetro. Estimated parameters and standard error of estimation $\left(\mathrm{s}_{\mathrm{y} . \mathrm{x}}\right)$ for the generalized height-diameter equations.

\begin{tabular}{|c|c|c|c|c|c|c|}
\hline \multirow{2}{*}{ Ecuación } & \multicolumn{5}{|c|}{ Parámetros estimados } & \multirow{2}{*}{$\begin{array}{l}s_{y, x} \\
(\mathrm{~m})\end{array}$} \\
\hline & $\hat{\beta}_{0}$ & $\hat{\beta}_{1}$ & $\hat{\beta}_{2}$ & $\hat{\beta}_{3}$ & $\hat{\beta}_{4}$ & \\
\hline E11 & 1,2118 & - & - & - & - & 1,72 \\
\hline E12 & $-0,00312$ & 10,6030 & - & - & - & 1,72 \\
\hline E13 & 10,5860 & 1,1520 & $-0,0044$ & - & - & 1,72 \\
\hline E14 & 5,9456 & 1,2716 & $-0,2033$ & 10,4786 & - & 1,72 \\
\hline E15 & 2,6386 & $-8,3120$ & $-0,9148$ & 0,03335 & - & 1,99 \\
\hline E16 & 0,3025 & 1,0028 & 0,44734 & $-0,11155 \mathrm{~ns}$ & - & 1,99 \\
\hline E17 & 1,0681 & 0,9302 & 2,49367 & $1,44663 \mathrm{~ns}$ & - & 1,82 \\
\hline E18 & 2,6037 & $-8,5107$ & $-0,92612$ & 0,03309 & 0,01033 & 1,99 \\
\hline E19 & 0,0105 & 0,000209 & $-0,000156$ & $-0,10270$ & $-1,80003$ & 1,74 \\
\hline
\end{tabular}

ns no significativo $(\alpha=0,05)$ 

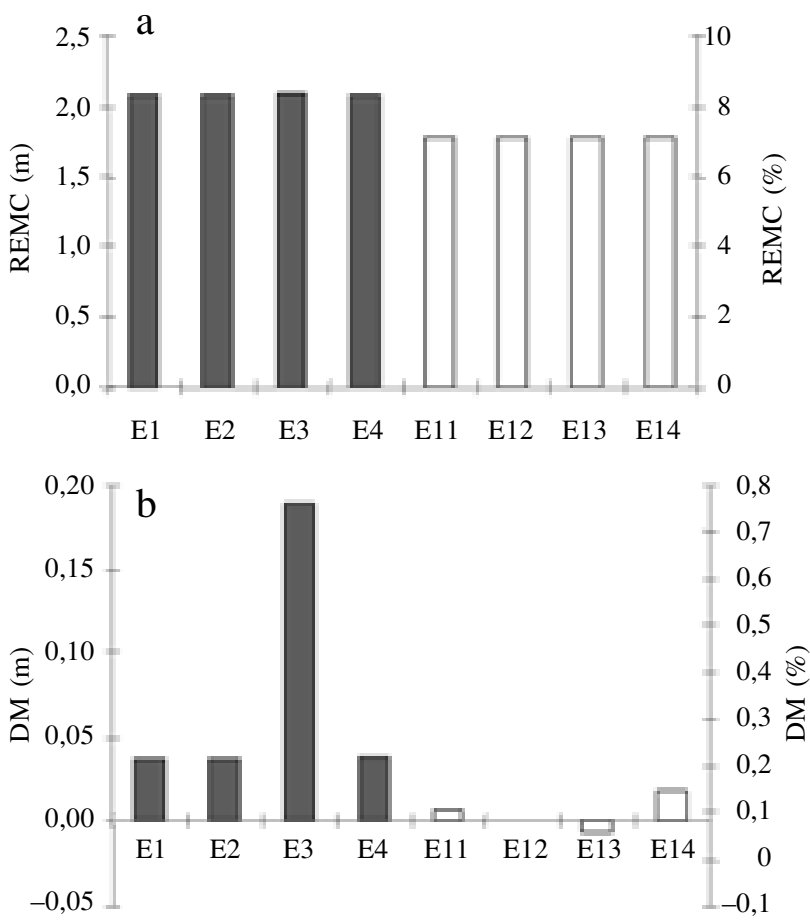

Ecuaciones locales

Ecuaciones generalizadas

Figura 2. Evaluación de la capacidad predictiva de las ecuaciones seleccionadas (a) precisión (REMC) y (b) sesgo (MD) dentro y entre estrategias de modelamiento.

Evaluation of selected equations (a) comparison of precisión (RMSE) and (b) comparison of bias within and between modeling approaches. para cada uno de los 105 rodales. Las diferencias observadas en la estimación de volúmenes y resultados obtenidos al aplicar una prueba $t$ para observaciones pareadas se presentan en el cuadro 8. Las diferencias media y máxima absoluta en volúmenes estimados no fueron mayores a $0,83 \mathrm{~m}^{3} \mathrm{ha}^{-1}$ y $11,83 \mathrm{~m}^{3} \mathrm{ha}^{-1}$, respectivamente. Sin embargo, la aplicación de una prueba $t$ para observaciones pareadas indicó que existen diferencias significativas al utilizar una ecuación local o generalizada de altura-diámetro en el cálculo de volúmenes totales, comerciales y productos $(P<0,05)$. El mismo test estadístico aplicado para cada uno de los productos detectó diferencias significativas sólo para AS02 $(P<0,05)$.

\section{DISCUSIÓN}

Ecuaciones locales y generalizadas. En base a medidas de bondad de ajuste, las ecuaciones locales no lineales de 2-parámetros fueron superiores a las ecuaciones de 3parámetros. Adicionalmente, la utilización de ecuaciones con 3-parámetros presentaron problemas de convergencia al emplear el algoritmo de minimización MARQUARDT. Se decidió aplicar otros algoritmos de minimización, pero se obtuvieron los mismos resultados. En la literatura no se ha informado sobre problemas de convergencia utilizando este tipo de ecuaciones (cuadro 3). Sin embargo, esto podría explicarse por el mayor número de observaciones utilizadas en el proceso de ajuste en estos estudios. Probablemente será necesario analizar en forma más detallada cada uno de los casos en los cuales se presentaron problemas de convergencia. De todas formas, no se

Cuadro 7. Número de veces en que cada ecuación fue asignada a un determinado ranking basado en su REMC para un total de 105 rodales.

Times receiving rankings for each of the selected equations for all of the 105 sampled stands, where ranking was by RMSE.

\begin{tabular}{|c|c|c|c|c|c|c|c|c|c|}
\hline \multirow{2}{*}{ Ecuación } & \multicolumn{8}{|c|}{ Ranking } & \multirow{2}{*}{$\begin{array}{c}\text { Ranking } \\
\text { medio }\end{array}$} \\
\hline & 1 & 2 & 3 & 4 & 5 & 6 & 7 & 8 & \\
\hline \multicolumn{10}{|l|}{ Local } \\
\hline E1 & 0 & 8 & 3 & 3 & 5 & 54 & 32 & 0 & 5,81 \\
\hline E2 & 2 & 4 & 5 & 4 & 29 & 16 & 23 & 22 & 5,90 \\
\hline E3 & 4 & 2 & 2 & 6 & 27 & 4 & 12 & 48 & 6,33 \\
\hline \multirow[t]{2}{*}{ E4 } & 6 & 0 & 3 & 4 & 32 & 19 & 26 & 15 & 5,78 \\
\hline & & & & & & & & Media & 5,96 \\
\hline \multicolumn{10}{|c|}{ Generalizada } \\
\hline E11 & 23 & 11 & 32 & 29 & 6 & 1 & 2 & 1 & 3,00 \\
\hline E12 & 24 & 27 & 22 & 17 & 4 & 2 & 6 & 3 & 2,95 \\
\hline E13 & 6 & 45 & 31 & 11 & 1 & 9 & 2 & 0 & 2,91 \\
\hline \multirow[t]{2}{*}{ E14 } & 41 & 9 & 7 & 33 & 2 & 1 & 3 & 9 & 3,06 \\
\hline & & & & & & & & Media & 2,98 \\
\hline
\end{tabular}


Cuadro 8. Diferencias observadas entre volúmenes $(V)$ estimados empleando una ecuación local y generalizada de alturadiámetro $\left(V_{\text {local }}-V_{\text {generalizada }}\right)$ y prueba $t$ para observaciones pareadas (105 rodales). (105 sampled stands).

Observed differences between stand volumes $(V)$ estimated using local and generalized equations $\left(V_{\text {local }}-V_{\text {generalized }}\right)$ and paired $t$-test

\begin{tabular}{|c|c|c|c|c|c|c|}
\hline \multirow{2}{*}{ Volúmenes } & \multicolumn{4}{|c|}{ Diferencias en volúmenes estimados $\left(\mathrm{m}^{3} \mathrm{ha}^{-1}\right)$} & \multirow{2}{*}{$|t|$} & \multirow{2}{*}{$\begin{array}{l}\text { Valor } \\
\text { de } P\end{array}$} \\
\hline & Media & Mínimo & Máximo & $\begin{array}{c}\text { Desviación } \\
\text { estándar }\end{array}$ & & \\
\hline Volumen total & $-0,83$ & $-11,83$ & 6,49 & 3,04 & 2,81 & 0,0059 \\
\hline Volumen comercial $^{\mathrm{a}}$ & $-0,79$ & $-10,75$ & 6,20 & 2,89 & 2,84 & 0,0054 \\
\hline Productos & $-0,75$ & $-11,23$ & 7,35 & 2,97 & 2,60 & 0,0107 \\
\hline AS01 & $-0,03$ & $-3,71$ & 2,20 & 1,05 & 0,29 & 0,7762 \\
\hline AS02 & $-0,53$ & $-5,86$ & 3,41 & 1,79 & 3,05 & 0,0029 \\
\hline Pulpa & $-0,19$ & $-4,09$ & 3,22 & 1,24 & 1,58 & 0,1177 \\
\hline
\end{tabular}

a Se considera un diámetro límite de utilización de $10 \mathrm{~cm}$

debiera aconsejar la implementación de ecuaciones locales de 3-parámetros en sistemas de procesamiento electrónico de inventarios. Al parecer la predicción de alturas totales utilizando un modelo de tipo no lineal y como variable predictora el DAP requiere considerar no más que dos parámetros. En esta investigación no se consideró la evaluación de ecuaciones de tipo lineal. Sin embargo, es posible asegurar que este tipo de ecuaciones no presentarían problemas en la estimación de parámetros al utilizar mímimos cuadrados.

Para todas aquellas ecuaciones ajustadas no fue necesaria la aplicación de factores de ponderación, debido a que no se detectaron problemas de heterogeneidad de varianza de los residuales. Por otro lado, en aquellos estudios en los cuales se han detectado problemas de heterocedasticidad, la utilización de factores de ponderación no han demostrado aumentar la capacidad predictiva de las ecuaciones de altura-diámetro (Curtis 1967, Leroy 1989, Zhang 1997).

En comparación a las ecuaciones locales, la totalidad de las ecuaciones generalizadas presentaron convergencia. El tiempo de convergencia fue dependiente del número de parámetros y complejidad de la ecuación. Durante el proceso de ajuste se determinó que dos de las ecuaciones (E16 y E17) presentaron parámetros que no fueron significativos. Sin embargo, no se realizaron esfuerzos para ajustar las ecuaciones sin estos parámetros. Las ecuaciones generalizadas con los menores valores de error estándar de estimación fueron seleccionadas para evaluar sus capacidades predictivas. Aun cuando la ecuación E19 presentó un ajuste aceptable, ésta requiere el uso de un mayor número de variables predictoras como son el número de árboles por hectárea $(N)$ y máximo $\operatorname{DAP}\left(d_{\max }\right)$.

Capacidad predictiva de ecuaciones locales versus ecuaciones generalizadas. En cuanto a medidas de precisión
(REMC) las ecuaciones generalizadas presentaron menores valores que las ecuaciones locales. La precisión observada para las ecuaciones generalizadas fue de $1,7 \mathrm{~m}$, que porcentualmente equivalen a un error medio aproximado de 7,2\% (figura 2a). Dentro de cada uno de los tipos de estrategias de modelamiento los valores observados para la REMC en las distintas ecuaciones fue parecido. Las mayores diferencias entre y dentro de las estrategias de modelamiento se observaron en los valores de sesgo (figura 2b). Nuevamente, los menores valores fueron observados al utilizar ecuaciones generalizadas. Todas las ecuaciones locales tendieron a subestimar, especialmente la ecuación E3. Para el caso de las ecuaciones generalizadas los valores de sesgos fueron cercanos a cero, y la única ecuación que presentó una leve sobreestimación fue la ecuación E13. Claramente las evaluaciones de precisión y sesgo indicaron que las ecuaciones generalizadas fueron superiores a las ecuaciones locales.

El cálculo de la REMC de cada una de las ecuaciones a nivel de rodal y la generación de un sistema de ranking permitió identificar las mejores ecuaciones dentro de cada una de las estrategias de modelamiento. En base al criterio utilizado las ecuaciones locales E1 y E4 y las ecuaciones generalizadas E12 y E13 fueron las mejores. Sin embargo, las ecuaciones generalizadas fueron notoriamente más precisas que las ecuaciones locales.

La estructura de las dos mejores ecuaciones locales es bastante similar, siendo la ecuación E1 una versión condicionada de la ecuación E4. Por otro lado, las variables auxiliares incluidas en ambas ecuaciones generalizadas (E12 y E13) corresponden al diámetro y altura total de los árboles dominantes. La incorporación de estas variables ratifica el efecto de la productividad de sitio sobre la relación altura-diámetro (figura 1b). Por lo tanto, la variabilidad de sitio existente en un rodal debe ser considerada en la predicción de alturas totales. 
Predicción de altura total y cálculo de volúmenes. La predicción de alturas totales utilizando una ecuación local o generalizada presentó un efecto significativo en el cálculo de volúmenes totales, comerciales y productos $(P<0,05)$. Se observó que las menores diferencias se obtuvieron al calcular volúmenes a nivel de productos. En dos de tres productos no exitieron diferencias significativas en la estimación de volúmenes al utilizar diferentes métodos de predicción de alturas totales. Sin embargo, debido a que las ecuaciones generalizadas presentan una mayor precisión en la estimación de alturas totales se podría inferir que las estimaciones volumétricas y de productos debieran ser también más precisas.

Una comparación de ambos métodos de predición de alturas totales debiera considerar la eficiencia para reducir costos operacionales de captura de datos y simplificación en el procesamiento de datos. En este contexto, el uso de una única ecuación generalizada reduciría el número de árboles muestras para la medición de alturas o al menos a igual número de árboles muestras permitiría mejorar la precisión en la predicción de alturas totales dentro de cada parcela (figura 2). Por ejemplo, la aplicación de E13 requeriría sólo la medición de alturas en árboles dominantes dentro de cada parcela de inventario, que además son aquellos árboles que presentan una mejor visibilidad. Si no se desea o cuenta con mediciones de alturas en cada parcela, se puede obtener una estimación indirecta de altura dominante utilizando una ecuación de índices de sitio. Este procedimiento asume que la edad del rodal y la calidad de sitio donde se encuentra la plantación son conocidos. Sin embargo, se debe considerar que este tipo de estimación afectaría la precisión en la estimación de alturas totales al emplear una ecuación generalizada en comparación a la medición directa de alturas (dominantes) en cada parcela.

Por otro lado, durante la etapa de procesamiento el uso de una única ecuación generalizada evitaría el ajuste de ecuaciones locales para cada rodal inventariado.

\section{CONCLUSIONES}

Las ecuaciones locales no lineales de altura-diámetro de 2-parámetros fueron superiores a las ecuaciones de 3-parámetros. Una evaluación de la capacidad predictiva indicó que las ecuaciones locales E1 y E4 y las ecuaciones generalizadas E12 y E13 fueron las mejores dentro de cada estrategia de modelamiento. Sin embargo, las ecuaciones generalizadas presentaron mayor precisión y menores sesgos que las ecuaciones locales.

La utilización de ecuaciones locales o generalizadas en el cálculo de volúmenes totales y comerciales indicaron diferencias significativas $(P<0,05)$. Sin embargo, debido a que las ecuaciones generalizadas presentan mayor precisión en la estimación de alturas se podría inferir que las estimaciones volumétricas y de productos debieran ser también más precisas.

\section{AGRADECIMIENTOS}

Los autores agradecen a Forestal Mininco S.A. por facilitar generosamente la información dasométrica requerida para el desarrollo de esta investigación.

\section{REFERENCIAS}

Allen PJ, NB Henry, P Gordon. 1992. Polynomial taper model for Queensland plantation hoop pine. Australian Forestry 55: 9-14.

Arabatzis AA, HE Burkhart. 1992. An evaluation of sampling methods and model forms for estimating height-diameter relationships in loblolly pine plantations. For. Sci. 38: 192-198.

Assman E. 1970. The principles of forest yield studies. Pergamon Press, Oxford. 506 p.

Avery TE, HE Burkhart. 2002. Forest measurements. Fifth Edition. McGraw-Hill. 456 p.

Bruce D, R Curtis, G Vancoevering. 1968. Development of a system of taper and volume tables for red alder. For. Sci. 14(3): 339-350.

Burkhart HE, RC Parker, MR Strub, RG Oderwald. 1972. Yield of old -field loblolly pine plantations. School of Forestry and Wildlife Resources, Virginia Polytechnic Institute and State University, Blacksburg, Publ. FWS-3-72.

Bi H, V Jurskis, J O'Gara. 2000. Improving height prediction of regrowth eucalypts by incorporating the mean size of site trees in a modified Chapman-Richards equation. Australian Forestry 63 (4): 255-264.

Cox F. 1994. Modelos parametrizados de altura. Informe de Convenio Investigación Interempresas. INFORA, Santiago. $28 \mathrm{p}$.

Curtis RO. 1967. Height-diameter and height-diameter-age equations for second growth Douglas-fir. For. Sci. 13(4): 365-375.

Dolph KL. 1989. Height-diameter equations for young-growth red fir in California and southern Oregon. Res. Note PSW408. Berkeley, CA: Pacific Southwest Forest and Range Experiment Station, Forest Service, U. S. Department of Agriculture.

Dorado FC, AD Ruiz González, JG Álvarez González. 2001. Modelización de la relación altura-diámetro para Pinus pinaster Ait. en Galicia mediante la función de densidad bivariante $\mathrm{S}_{\mathrm{BB}}$. Investigación Agraria: Sistema de Recursos Forestales 10(1): 111-125.

Eerikäinen K. 2003. Predicting the height-diameter pattern of planted Pinus kesiya stands in Zambia and Zimbabwe. For. Ecol. Manage. 175: 355-366.

Epstein R, E Nieto, A Weintraub, P Chevalier, J Gabarró. 1999. A system for the design of short term harvesting strategy. European Journal of Operational Research 119: 427-439.

Fang Z, RL Bailey. 1998. Height-diameter models for tropical forests on Hainan Island in southern China. For. Ecol. Manage. 110: 315-327.

Flewelling JW, R Jong. 1994. Considerations in simultaneous curve fitting for repeated height-diameter measurements. Can. J. For. Res. 24: 1408-1414. 
Gadow Kv, G Hui. 1999. Modeling forest development. Kluwer Academic Publishers. 228 p.

Gaffrey D. 1988. Forstamts- und bestandesindividuelles Sortimentierungsprogramm als Mittel zur Planung, Aushaltung und Simulation. Diplomarbeit, Göttingen, Alemania, Forstliche Fakultät, Universität Georg-August. 86 p.

García O. 1974. Ecuación altura-diámetro para pino insigne. Nota Técnica № 19, Instituto Forestal, Chile. 15 p.

García O. 1996. Toward new site index curves for Douglas-fir in the Netherlands. Working Paper, Royal Veterinary and Agricultural University, Department of Economics and Natural Resources, Unit of Forestry. 11 p.

Hökka H. 1997. Height-diameter curves with random intercepts and slopes for trees growing on drained peat lands. For. Ecol. Manage. 97: 63-72.

Huang S, D Price, S Titus. 2000. Development of ecoregionbased height-diameter models for white spruce in boreal forests. For. Ecol. Manage. 129: 125-141.

Huang S, SJ Titus. 1994. An age-independent individual tree height prediction model for boreal spruce-aspen stands in Alberta. Can. J. For. Res. 24: 1295-1301.

Huang S, SJ Titus, DD Wiens. 1992. Comparison of nonlinear height-diameter functions for major Alberta tree species. Can. J. For. Res. 22: 1297-1304.

Hui G, Kv Gadow. 1993. Zur Entwicklung von Einheitshöhenkurven am Beispiel der Baumart Cunninghamia lanceolata. Allgemeine Forst- und Jagdzeitung 164(12): 218-220

Krumland B, L Wensel. 1988. A generalized height-diameter equation for Coastal California Species. W. J. Appl. For. 3(4): 113-115.

Lappi J. 1997. A longitudinal analysis of height-diameter curves. For. Sci. 43(4): 555-570.

Larsen DR, DW Hann. 1987. Height-diameter equations for seventeen tree species in southwest Oregon. Forest Research Laboratory. Oregon State University, Corvallis. Research Bulletin 49. 16 p.

Leroy D. 1989. Height-diameter equations for young-growth red fir in California and the southern Oregon. Research Note PSW-408, USDA Pacific Southwest Forest and Range Experiment Station. 4 p.

López Sánchez CA, J Gorgoso Varela, F Castedo Dorado, A Rojo Alboreceda, R Rodríguez Soalleiro, JG Álvarez González, F Sánchez Rodríguez. 2003. A height-diameter model for Pinus radiata D. Don in Galicia (Northwest Spain). Ann. For. Sci. 60: 237-345.

Lynch T, P Murphy. 1995. A compatible height prediction and projection system for individual trees in natural, evenaged shortleaf pine stands. For. Sci. 41(1): 194-209.

Martin F. 1993. Construcción de modelos de razón. Tesis de Pregrado, Facultad de Ciencias Forestales, Universidad Austral de Chile. 48 p.

Martin F, J Flewelling. 1998. Evaluation of tree height prediction models for stand inventory. W. J. Appl. For. 13(4): 109-119.

Moore JA, L Zhang, D Stuck. 1996. Height-diameter equations for ten tree species in the Inland Northwest. W. J. Appl. For. 11(4): 132-137.

Myers RH. 1990. Classical and modern regression with applications. $2^{\text {nd }}$ Edition, Boston, PWS-Kent, Publishing Company. $488 \mathrm{p}$.
Nagel J. 1991. Einheitshöhenkurvenmodell für Roteiche. Allgemeine Forst und Jagdzeitung 162(1): 16-48.

Nash AJ. 1965. Statistical techniques in forestry. Lucas Brothers Publishers, Columbia, Missouri. 146 p.

Omule SA, RN MacDonald. 1991. Simultaneous curve for repeated height-diameter measurements. Can. J. For. Res. 21: 1418-1422.

Parresol BR. 1992. Baldcypress height-diameter equations and their prediction confidence intervals. Can. J. For. Res. 22: 1429-1434.

Peng C, L Zhang, J Liu. 2001. Developing and validating nonlinear height-diameter models for major tree species of Ontario's Boreal Forests. N. J. Appl. For. 18: 87-94.

Prodan M. 1965. Holzmesslehre. J.D. Sauerländer's Verlag, Frankfurt am Main. 644 p.

Prodan M, R Peters, F Cox, P Real. 1997: Mensura Forestal. Instituto Interamericano de Cooperación para la Agricultura (IIAC)/Deutsche Gesellschaft für Technische Zusammenarbeit (GTZ) GmbH, San José, Costa Rica. 561 p.

SAS Institute Inc. 1999. SAS User' s Guide: SAS STAT.Version 8. SAS Institute Inc., Cary. NC. 3.844 p.

Schröder J, JC Álvarez González. 2001. Comparing the performance of generalized height-diameter equations for Maritime pine in Northwestern Spain. Forstwissenschaftliches Centralblatt 120: 18-23.

Sloboda B, D Gaffrey, N Matsumura. 1993. Regionale und lokale Systeme von Höhenkurven für gleichaltrige Waldbestände. Allgemeine Forst und Jagdzeitung 164(12): 225-228.

Soares P, M Tomé. 2002. Height-diameter equation for first rotation eucalypt plantations in Portugal. For. Ecol. Manage. 166: 99-109.

Tewari VP, Kv Gadow. 1999. Modelling the relationship between tree diameters and heights using $\mathrm{S}_{\mathrm{BB}}$ distribution. For. Ecol. Manage. 119: 171-176.

van Laar C. 1986. Die Güte der Anpassung linearer Modelle zur Darstellung der Bestandeshöhenkurve. Forstarchiv 57: 134-137.

van Laar A, A Akça. 1997. Forest Mensuration. Cuvillier Verlag, Göttingen. 418 p.

Wang C, DW Hann. 1988. Height-diameter equations for sixteen tree species in the Central Western Willamette Valley of Oregon. Forest Research Lab., College of Forestry, Oregon State University. 7 p.

Wenk G, V Antanaitis, S Smelko. 1990. Waldertragslehre. Deutschland. Deutscher Landwirtschaftsverlag. 448 p.

Woollons RC. 2003. Examination of mean top height definitions and the height estimation equations for Pinus radiata in New Zealand. NZ Journal of Forestry 48: 15-18.

Wykoff WR, NL Crookston, AR Stage. 1982. User's guide to the stand prognosis model. Gen. Tech. Rep INT-133. Ogden, UT: Intermountain Forest and Range Station, Forest Service, U.S. Department of Agriculture. 112 p.

Yang RC, A Kozak, JHG Smith. 1978. The potential of Weibull-type functions as a flexible growth curve. Can. J. For. Res. 8: 424-431.

Yuancai L, BR Parresol. 2001. Remarks on height-diameter modeling. USDA Forest Service, Res. Note RN-SRS-10. 5 p.

Zeide B. 1989. Accuracy of equations describing diameter growth. Can. J. For. Res. 19: 1283-1286. 
Zhang L, JA Moore, JD Newberry. 1995. Evaluation of the prediction performance for eight nonlinear height-diameter equations. Poster presented at the Resources Measurement subject area at the SAF National Convention held in Portland, Main, October 28-November 1. 1995, 447-448.
Zhang L. 1997. Cross-validation of non-linear growth functions for modelling tree height-diameter relationships. Annals of Botany 79: 251-257.

Zhang L, C Peng, S Huang, X Zhou. 2002. Development and evaluation of ecoregion-based jack pine height-diameter models for Ontario. Forestry Chronicle 78(40): 530-538.

Recibido: 13.03.05

Aceptado: 09.03.06 